\title{
Optimal dynamic vibration absorber design for minimizing the band-averaged input power using the residue theorem
}

\author{
R. D’Amico*, K. Koo, C.C. Claeys, B. Pluymers, W. Desmet \\ KU Leuven, Department of Mechanical Engineering, Celestijnenlaan 300 B, 3001, \\ Leuven, Belgium.
}

\begin{abstract}
This paper deals with an efficient strategy to improve the vibro-acoustic behavior of a structure over frequency bands. Genetic Algorithms are used to identify the optimal resonance frequency and location of Dynamic Vibration Absorbers (DVAs) which minimize the band-averaged input power into a plate, leading to an indirect reduction of the radiated acoustic power and global vibration. Instead of classic numerical quadrature schemes, the residue theorem is used to evaluate the band-averaged input power. This results into a considerable reduction of computational effort, as it requires only few function evaluations at complex frequencies, regardless of the analyzed bandwidth. The structural response is simulated by using the Wave Based Method (WBM). Besides an increased convergence rate as compared to classical element-based techniques, the WBM is also free in determining the optimal position of the DVAs, not restricting it to nodal grid locations. Moreover, when point connections are taken into account, only a small part of the WB matrices needs to be recomputed at each iteration, resulting in a strong reduction of the computation time. Numerical examples illustrate the benefits and the efficiency of the proposed optimization strategy.
\end{abstract}

Keywords: Wave Based Method, input power, genetic algorithms, dynamic vibration absorbers, band-averaging

*Corresponding author. Tel. +32 163727 81; Fax: +32 16322987.

Email address: roberto.damico@kuleuven. be (R. D'Amico) 


\section{Introduction}

The control of the vibration of a structure and its radiated noise are key aspects to consider when designing a mechanical system. This can be achieved by changing the properties of the system, i.e. by varying its stiffness, mass or damping, or by actively acting on the oscillation by introducing external influences. The former strategy is called passive vibration control, and among the numerous passive devices, Dynamic Vibration Absorbers (DVA) are widely used $[1,2,3]$.

A DVA is generally modeled as a mass-spring-damper resonator, and, due to the light-weight properties, it can be easily installed on engineering structures, such as vehicles and industrial machineries [4]. The ability to absorb energy from the host structure is maximal at the resonance frequency of the DVA and is also related to its damping coefficient. Conventional approaches suggest to design the DVA such that it can abate the vibration level of one mode. This is sometimes done by considering the behavior of the bare structure, rather than a coupled fluid-structure model. However, this procedure is not always very effective. In fact, when the noise at one tone is reduced, the noise at other frequencies may increase, resulting into a global worsening of the perceived sound. Furthermore, DVAs can lose their efficiency when small changes occur with respect to the nominal host system or to the original resonator design. In real-life applications, operating conditions or manufacturing processes inevitably introduce uncertainty and variability in the model description, causing possible changes in the forcing frequency or in the system response. These reasons motivate the strong need for DVA design procedures to improve the vibrational behavior over a band rather than at a single target frequency. In this regard, optimization techniques are very helpful to identify the best performing configuration. A procedure is proposed in ref. [5], where the properties of the DVA are optimized by solving a non-linear constraint optimization problem. The behavior of the structure improves significantly over the band of interest, and the final design is proven to be robust against variations of the DVA properties. A very recent approach [6] is based on the minimization of the worst-case gain related to the frequency band of interest; Genetic Algorithms (GAs) [7] are used to perform the optimization. Also in ref. [8], GAs are used to optimize the parameters of DVAs and Helmholtz resonators, where the objective function is the acoustic potential energy within a payload fairing. Due to the large number of passive systems, the energy is smeared out over a broad frequency 
range.

An alternative way of creating a stop-band behavior is proposed in ref. [9], where a grid of resonators properly distributed over a panel allows the global vibration level to be significantly attenuated over a band of frequencies. Instead of determining the properties by means of an optimization procedure, the ratio between the frequency of the resonator and the propagating wave frequency of the unit cell is proven to be key for the efficiency of the grid.

The efficient algorithm proposed in this paper allows improving the DVA design over a band of frequencies. Instead of using global displacements or acoustic-related quantities, the input power into the structure is employed as an objective function. The behavior of the optimal configuration shows a strong improvement over the band of interest, although the radiated sound and global displacement are not minimized in general. On the other hand, significant computational savings are possible. In fact, when a band is targeted, rather than a single frequency, employing classic quadrature to evaluate the average may lead to a dramatic increase of computation time. As proposed in ref. [10, 11], a few computation at complex frequencies can replace a refined quadrature integration when evaluating the input power, still providing accurate band computations.

The choice of the band-averaged input power as an objective function for optimal DVA design, together with the aforementioned approach to significantly speed up the calculation, constitutes the core of the paper and leads to a very efficient and flexible strategy for DVA design that can be easily extended to problems of larger size.

In the proposed applications, GAs are used for the optimization process, and the Wave Based Method (WBM) $[12,13]$ is employed to simulate the dynamic behavior of the plate. Compared to traditional Finite Element Method (FEM) [14], the WBM shows a higher convergence rate and does not restrict the DVA position to nodal locations. Moreover, when dealing with point connections, only a few rows and columns have to be added to the system of equations of the bare structure. These advantageous features perfectly fit the framework of an optimization scheme.

The vibration reduction of a rectangular plate excited by a point force is investigated in two different scenarios. In the first case, one DVA is optimized such that it can minimize the input power over one band. In the second case, two DVAs are used to minimize the input power over two frequency bands. In all cases, minimizing the band-averaged input power leads to a reduction 
of global vibration and radiated acoustic power. Additionally, the obtained designs are proven to be robust against variations in the DVA parameters. The computational performance is assessed, in order to show the significant advantages of the use of the residue theorem against classic quadrature techniques.

The paper is structured as follows. In sec. 2, theoretical aspects related to the problem formulations and the WBM are discussed. The strategy presented in [10] is briefly recalled in sec. 3. Section 4 is dedicated to the description of the optimization procedure, which is adopted for the application cases in sec. 5. Conclusions are drawn in sec. 6.

\section{Theoretical aspects and numerical methodologies}

The focus in this paper is on the acoustic radiation from a baffled thin plate. In the following sections, the numerical approaches to predict the response of the uncoupled vibro-acoustic system are presented. In particular, to solve the structural problem the WBM is used with its extension to point connections [15]. On the other hand, the Rayleigh integral is used to compute the acoustic power radiated from the plate [16].

Steady-state conditions are considered and the time convention is chosen such that the oscillation is described by $\mathrm{e}^{\mathrm{i} \omega t}$, where $\mathrm{i}$ is the imaginary unit, $\omega$ is the angular frequency and $t$ indicates the time. The problem geometry is depicted in fig. 1.

\subsection{Theory}

For thin plates and low frequencies, the effects of rotary inertia and shear deformation can be neglected. This leads to the Kirchhoff theory [17], for which the out-of-plane displacements $w$ of a plate in bending are governed by the following partial differential equation,

$$
\nabla^{4} w(\mathbf{x})-k_{b}^{4} w(\mathbf{x})=\frac{1}{D} F \delta\left(\mathbf{x}, \mathbf{x}_{f}\right)+\frac{1}{D} \sum_{i=1}^{n_{p c}} F_{p c, i} \delta\left(\mathbf{x}, \mathbf{x}_{p c, i}\right), \quad \mathbf{x} \in \Omega_{\mathrm{p}}
$$

where $\nabla^{4}=\frac{\partial^{4}}{\partial x^{4}}+2 \frac{\partial^{4}}{\partial x^{2} \partial y^{2}}+\frac{\partial^{4}}{\partial y^{4}}$. The plate bending wavenumber, $k_{b}$, and bending stiffness, $D$, are defined as,

$$
k_{b}=\sqrt[4]{\frac{\rho_{p} h \omega^{2}}{D}}
$$




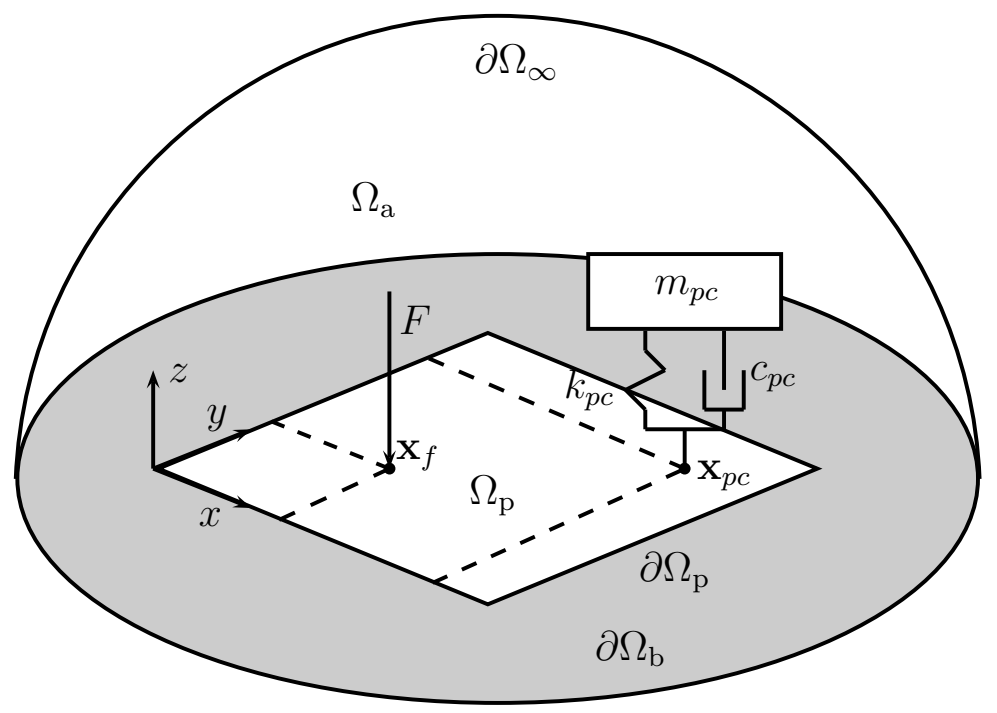

Figure 1: Problem geometry.

$$
D=\frac{E(1+\mathrm{i} \eta) h^{3}}{12\left(1-\nu^{2}\right)}
$$

where $\rho_{p}$ is the material density, $h$ is the plate thickness, $E$ is the Young modulus, $\eta$ is the material damping factor and $\nu$ is the Poisson ratio. The symbol $F$ represents the complex amplitude of the harmonic point force applied at $\mathbf{x}_{f}$, while $F_{p c, i}$ is the force exerted by the $i$-th point-connected DVA. $\delta$ indicates the Dirac delta. The plate surface coincides with the twodimensional domain $\Omega_{\mathrm{p}}$ of boundary $\partial \Omega_{\mathrm{p}}$.

As eq. (1) is a fourth-order partial differential equation, two boundary conditions have to be imposed over each partition of the boundary $\partial \Omega_{\mathrm{p}}$. These can be either kinematic conditions, i.e. for clamped edges, or mechanical boundary conditions, i.e. for free or loaded edges. Finally, a mixed type of boundary conditions can be defined by using the residue of the displacement and bending moment at point $\mathbf{x} \in \partial \Omega_{\mathrm{p}}$. These can be expressed as follows,

$$
\begin{gathered}
R_{w}(\mathbf{x})=w(\mathbf{x})-\bar{w}(\mathbf{x})=0, \\
R_{m}(\mathbf{x})=\mathcal{L}_{m}[w(\mathbf{x})]-\bar{m}(\mathbf{x})=0,
\end{gathered}
$$

where $\bar{w}$ is the prescribed displacement, $\bar{m}$ the prescribed bending moment 
and $\mathcal{L}_{m}$ is the generalized bending moment operator,

$$
\mathcal{L}_{m}=-D\left(\frac{\partial^{2}}{\partial n^{2}}+\nu \frac{\partial^{2}}{\partial s^{2}}\right)
$$

with $n$ and $s$ the in-plane normal and tangential directions of the plate boundary $\partial \Omega_{\mathrm{p}}$. In case the plate is simply supported, the prescribed displacements and bending moments are zero.

In the following applications, the thin baffled plate is immersed into a fluid. Under steady-state conditions, the acoustic problem is governed by the Helmholtz equation [18]

$$
\nabla^{2} p(\mathbf{x})+k_{a}^{2} p(\mathbf{x})=0, \quad \mathbf{x} \in \Omega_{\mathrm{a}}
$$

where $p$ is the acoustic pressure, $k_{a}$ is the acoustic wavenumber $\frac{\omega}{c}$, with $c$ the speed of sound in the media of density $\rho_{a}$. The three-dimensional semiunbounded fluid is represented by the domain $\Omega_{\mathrm{a}}$. The boundary can be split in three non-overlapping regions, $\Omega_{\mathrm{p}} \cup \partial \Omega_{\mathrm{b}} \cup \partial \Omega_{\infty}$, where $\Omega_{\mathrm{p}}$ is the fluid-plate interface boundary, $\partial \Omega_{\mathrm{b}}$ is the infinite baffle and $\partial \Omega_{\infty}$ is the boundary at infinity, on which the Sommerfeld condition is applied.

The interaction between fluid and structure can be modeled in two ways. When the structure has high stiffness and is in contact with a low-density fluid, the interaction between the two is weak and can be neglected. The two domains can be considered separately and each one serves as an exterior excitation for the other. If the elastic structure is immersed in a high-density fluid, the two are strongly coupled and mutually interacting with each other. In this case, they have to be modeled as a coupled vibro-acoustic system. Although this approximation is valid in any case, it is more computationally demanding, due to coupling terms taken into account.

In the following case, the plate presents a high stiffness and the fluid has low density, thus it is reasonable to assume that the mutual interaction is weak and negligible. Under this assumption, the problem can be treated as uncoupled, and the normal plate velocity distributions are independent of the surface pressures.

A reflecting boundary condition is applied over the baffle $\partial \Omega_{\mathrm{b}}$, while on the boundary $\Omega_{\mathrm{p}}$, a velocity boundary condition is applied from the structural vibration. This is formalized as follows,

$$
\mathcal{L}_{v}[p(\mathbf{x})]=\mathrm{i} \omega w(\mathbf{x}),
$$

where $\mathcal{L}_{v}$ is the normal velocity operator $\mathcal{L}_{v}(\bullet)=\frac{\mathrm{i}}{\rho_{a} \omega} \frac{\partial \bullet}{\partial n}$.

The acoustic radiated power can be used as a measure of the noise emitted 
from a structure. Supposing that the surface of the plate is part of an infinite plane, the pressure at a point $\mathbf{x} \in \Omega_{\mathrm{a}}$ can be computed by means of the Rayleigh integral,

$$
p(\mathbf{x})=\frac{\mathrm{i} \omega \rho_{\mathrm{a}}}{2 \pi} \int_{\Omega_{\mathrm{p}}} v_{n}(\mathbf{y}) \frac{\mathrm{e}^{-\mathrm{i} k_{\mathrm{a}} R}}{R} \mathrm{~d} \Omega_{\mathrm{p}}(\mathbf{y})
$$

where $R=|\mathbf{x}-\mathbf{y}|$, and $v_{n}$ is the normal velocity. The acoustic power radiated from the vibrating plate can be computed by integrating the acoustic intensity over the surface $\Omega_{\mathrm{p}}$, obtaining the following,

$$
W_{\mathrm{a}}=\frac{1}{2} \int_{\Omega_{\mathrm{p}}} \operatorname{Re}\left\{p(\mathbf{x}) \cdot v_{n}^{*}(\mathbf{x})\right\} \mathrm{d} \Omega_{\mathrm{p}}(\mathbf{x})
$$

which has been integrated by using 400 Gauss points over the surface $\Omega_{\mathrm{p}}$ [19].

\subsection{The $W B M$ for plate bending problems}

The WBM belongs to the family of Trefftz approaches [20], in which the field variables are expanded in terms of wave functions, which satisfy a priori the governing partial differential equation (1). Consequently, when a point excitation is present, the out-of-plane displacement field $w$ of the plate is approximated by the following expansion of wave functions,

$$
w(\mathbf{x}) \approx \hat{w}(\mathbf{x})=\sum_{b=1}^{n_{b}} c_{b} \Psi_{b}(\mathbf{x})+\hat{w}_{f}(\mathbf{x})=\mathbf{\Psi}(\mathbf{x}) \cdot \mathbf{c}+\hat{w}_{f}(\mathbf{x}),
$$

with $\Psi$ and $\mathbf{c}$ vectors containing $n_{b}$ wave functions $\Psi_{b}$, and the corresponding contribution factors $c_{b}$. The function $\hat{w}_{f}$ is the particular solution of eq. (1) subject to the force of amplitude $F$ and is defined as

$$
\hat{w}_{f}(\mathbf{x})=-\frac{\mathrm{i} F}{8 k_{b}^{2} D}\left[H_{0}^{(2)}\left(k_{b} r_{f}\right)-H_{0}^{(2)}\left(-\mathrm{i} k_{b} r_{f}\right)\right]
$$

where $r_{f}$ is the Euclidean distance between the excitation point $\mathbf{x}_{f}$ and the generic point $\mathbf{x} . H_{0}^{(2)}$ is the Hankel function of the second kind with order zero. The wave functions $\Psi_{b}$ have mathematical form

$$
\begin{array}{ll}
\Psi_{b_{1}}(\mathbf{x})=\cos \left(k_{b_{1, x}} x\right) \mathrm{e}^{-\mathrm{i} k_{b_{1, y}} y} & b_{1}=0,1, \ldots, n_{b_{1}}, \\
\Psi_{b_{2}}(\mathbf{x})=\mathrm{e}^{-\mathrm{i} k_{b_{2, x}} x} \cos \left(k_{b_{2, y}} y\right) & b_{2}=0,1, \ldots, n_{b_{2}} .
\end{array}
$$


This set of functions has been proven to be sufficient for convergence of the WBM, provided that the domain is convex [12].

From an infinite number of wave functions, a truncated set must be selected based on the dimension of the smallest rectangle circumscribing the plate geometry, of which the dimensions are $L_{x} \times L_{y}$. The first wavenumber components are chosen such that an integer number of half wavelengths equals the dimension of the rectangular box in the corresponding direction,

$$
\begin{aligned}
& k_{b_{1, x}}=\frac{b_{1} \pi}{L_{x}}, \\
& k_{b_{2, y}}=\frac{b_{2} \pi}{L_{y}} .
\end{aligned}
$$

The other wavenumber components are calculated from the bending wave number $k_{b}$ corresponding to the considered frequency,

$$
\begin{aligned}
& k_{b_{1, y}}=\left\{\begin{array}{l} 
\pm \sqrt{k_{b}^{2}-k_{b_{1, x}}^{2}} \\
\pm \mathrm{i} \sqrt{k_{b}^{2}+k_{b_{1, x}}^{2}}
\end{array},\right. \\
& k_{b_{2, x}}=\left\{\begin{array}{l} 
\pm \sqrt{k_{b}^{2}-k_{b_{2, y}}^{2}} \\
\pm \mathrm{i} \sqrt{k_{b}^{2}+k_{b_{2, y}}^{2}}
\end{array}\right.
\end{aligned}
$$

The number of bending wave functions $n_{b}$, that are included in the field variable expansion (11) is related to the frequency of analysis and the dimension of the enclosing bounding box,

$$
n_{b}=4\left(n_{b_{1}}+1\right)+4\left(n_{b_{2}}+1\right)
$$

where $n_{b_{1}} / L_{x} \approx n_{b_{2}} / L_{y} \geq T k_{b} / \pi$, with $n_{b_{1}}$ and $n_{b_{2}}$ integer truncation numbers and $T$, a user defined truncation parameter [21].

The boundary conditions are enforced by using a Galerkin weighted residual formulation, thus the residuals are orthogonalized with respect to a weighting function $\tilde{w}$, expanded in terms of the same set of wave functions used in the field variable expression (11). In case of a simply supported plate, the residual formulation results in the following expression

$$
\int_{\partial \Omega_{\mathrm{p}}} \mathcal{L}_{Q}[\tilde{w}(\mathbf{x})] R_{w}(\mathbf{x}) \mathrm{d} \partial \Omega_{\mathrm{p}}-\int_{\partial \Omega_{\mathrm{p}}} \mathcal{L}_{\theta_{n}}[\tilde{w}(\mathbf{x})] R_{m}(\mathbf{x}) \mathrm{d} \partial \Omega_{\mathrm{p}}=0
$$


where $\mathcal{L}_{Q}$ and $\mathcal{L}_{\theta}$ are respectively the generalized shear force and rotation operators defined as follows,

$$
\begin{aligned}
\mathcal{L}_{Q} & =-D \frac{\partial}{\partial n}\left[\frac{\partial^{2}}{\partial n^{2}}+(2-\nu) \frac{\partial^{2}}{\partial s^{2}}\right] \\
\mathcal{L}_{\theta} & =-\frac{\partial}{\partial n} .
\end{aligned}
$$

This results into a system of $n_{b}$ equations in $n_{b}$ unknowns,

$$
\mathrm{Ac}=\mathbf{f}
$$

of which the system matrix $\mathbf{A}$ is complex, fully populated and frequency dependent. By solving the system of equations, the contribution factors $\mathbf{c}$ can be evaluated and substituted in the field expansion (11), to compute the system response at point $\mathbf{x}$.

For the sake of brevity, the WBM formulation is here presented only for the case of simply supported boundary conditions. The reader is referred to ref. [15, 22] for a more complete and general formulation in acoustics and structural dynamics.

\subsection{Extension of the WBM to point connections}

According to the formulation presented in ref. [15], the effect of $n_{p c}$ DVAs now is included into the WB formulation. The variable expansion (11) results in the following expression,

$$
\hat{w}(\mathbf{x})=\mathbf{\Psi}(\mathbf{x}) \cdot \mathbf{c}+\hat{w}_{f}(\mathbf{x})+\sum_{i=1}^{n_{p c}} \hat{w}_{p c, i}(\mathbf{x}) .
$$

The symbol $\hat{w}_{p c, i}$ denotes the particular solution for a force acting on the plate,

$$
\hat{w}_{p c, i}(\mathbf{x})=-\frac{\mathrm{i} f_{p c, i}(\omega) w_{p c, i}}{8 k_{b}^{2} D}\left[H_{0}^{(2)}\left(k_{b} r_{p c, i}\right)-H_{0}^{(2)}\left(-\mathrm{i} k_{b} r_{p c, i}\right)\right]
$$

where $r_{p c, i}$ is the Euclidean distance between $\mathbf{x}$ and the location of the $i$ th DVA, $\mathbf{x}_{p c, i}$, and $f_{p c, i}(\omega)$ represents the force-displacement relation with mathematical expression,

$$
f_{p c, i}(\omega)=\frac{F_{p c, i}}{w_{p c, i}}=\frac{m_{p c, i} \omega^{2}\left(k_{p c, i}+\mathrm{i} \omega c_{p c, i}\right)}{k_{p c, i}+\mathrm{i} \omega c_{p c, i}-m_{p c, i} \omega^{2}}
$$


where $k_{p c, i}$ is the stiffness of the $i$-th DVA, $m_{p c, i}$ is its mass and $c_{p c, i}$ the damping coefficient. The introduction of the particular solution (24) into the residual formulation (19) leads to a system of $\left(n_{b}\right) \times\left(n_{b}+n_{p c}\right)$ equations, to which $n_{p c}$ auxiliary equations are added as follows,

$$
w_{p c, i}\left(\mathbf{x}_{p c, i}\right)=\Psi\left(\mathbf{x}_{p c, i}\right) \cdot \mathbf{c}+\hat{w}_{f}\left(\mathbf{x}_{p c, i}\right)+\sum_{i=1}^{n_{p c}} \hat{w}_{p c, i}\left(\mathbf{x}_{p c, i}\right) .
$$

The system of equations now has the form

$$
\left[\begin{array}{ll}
\mathbf{A} & \mathbf{B} \\
\mathbf{C} & \mathbf{D}
\end{array}\right]\left\{\begin{array}{c}
\mathbf{c} \\
\mathbf{w}_{p c}
\end{array}\right\}=\left\{\begin{array}{c}
\mathbf{f} \\
\mathbf{f}^{\prime}
\end{array}\right\}
$$

where the vector $\mathbf{w}_{p c}$ is composed of the displacements at each point connection location. For the sake of brevity, matrices $\mathbf{B}, \mathbf{C}, \mathbf{D}$ and vector $\mathbf{f}^{\prime}$ are not reported in detail here. The reader is referred to ref. [15] for their complete expressions. However, it is worth noting that the inclusion of point connections does not influence the main matrix, $\mathbf{A}$, and only requires the addition of $n_{p c}$ columns and $n_{p c}$ rows to the system. As a consequence, to analyze different configurations, the main system $\mathbf{A}$ is not recomputed. Moreover, since the WBM matrices are generally very small, preprocessing the WBM matrices is more expensive than solving the system. Hence, this constitutes an advantageous property of the WBM for procedures which require iterations, such as optimization strategies and Monte Carlo simulations [15].

\section{Evaluation of band-averaged input power}

The power injected by the force $F$ and averaged over the band $\left[\omega_{0}-\right.$ $\left.\omega_{C}, \omega_{0}+\omega_{C}\right]$ can be evaluated as follows,

$$
\left\langle P_{\text {in }}\right\rangle_{W}=\frac{1}{2} \int_{-\infty}^{+\infty} W(\omega) \operatorname{Re}\left\{\mathrm{i} \omega w\left(\mathbf{x}_{f}, \omega\right) F^{*}\right\} \mathrm{d} \omega
$$

where $W(\omega)$ is defined as a rectangular window centered at $\omega_{0}$ and of halfwidth $\omega_{C}$. Integral (28) can be estimated by using classic quadrature techniques [23], although a refined sampling scheme is required to obtain accurate approximations, especially when the input mobility is highly oscillating over the interval of interest. Recently, the residue theorem [24] has been used as an alternative to numerical quadrature for computing band-averaged input 
power $[10,11]$. Instead of using a rectangular window, the square magnitude of a Butterworth filter,

$$
B(\omega)=\frac{A}{1+\left(\frac{\omega-\omega_{0}}{\omega_{C}}\right)^{2 n}},
$$

weighs the input power in eq. (28). The letter $A$ denotes the amplification factor of the filter and is equal to $n \sin (\pi / 2 n) / \pi \omega_{C}$. As the order $n$ increases, the shape of function $B$ better resembles an ideal rectangular window. This is illustrated in fig. 2, for orders 4,8 and 20 , and $A=1$. For a filter of order $n, 2 n$ poles are located in the complex frequency $z$ plane at the following positions,

$$
z_{k}=\omega_{0}+\omega_{C} \mathrm{e}^{-\mathrm{i} \theta_{k}}
$$

where

$$
\theta_{k}=\frac{\pi}{2 n}(1+2 k)
$$

The value $k$ is an integer which defines the position of a pole. When $0 \leq$ $k \leq n-1$, the $k$-th pole is located in the lower complex half plane; when $n \leq k \leq 2 n-1$, the $k$-th pole is located in the upper complex half plane. To clarify the notation, $z$ is used when the frequency value may be complex, while $\omega$ is used when the value is real.

The use of the residue theorem allows for computing integral (28) in a very

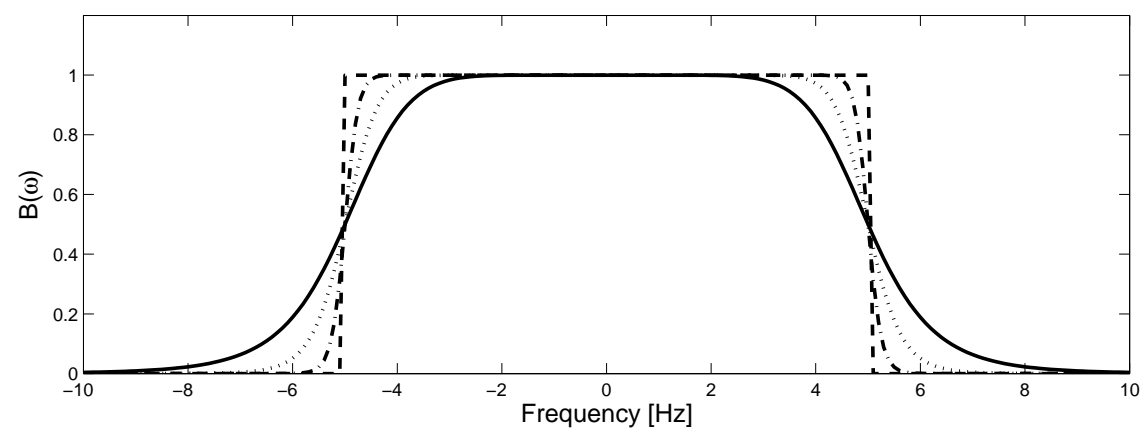

Figure 2: Comparison between a rectangular window (dashed line) and function $B(\omega)$ of order 4 (solid line), 8 (dotted line) and 20 (mixed line). The window is centered at $0 \mathrm{~Hz}$ and has a cut-off frequency equal to $5 \mathrm{~Hz}$.

straightforward manner and, as a result, the Butterworth-weighted frequency 
averaged input power into a structure can be evaluated by means of the following expression,

$$
\left\langle P_{\mathrm{in}}\right\rangle_{B}=-\frac{1}{2} \frac{\pi A \omega_{C}}{n} \operatorname{Re}\left\{F^{*} \sum_{k=0}^{n-1} z_{k} w\left(\mathbf{x}_{f}, z_{k}\right) e^{-\mathrm{i} \theta_{k}}\right\},
$$

which is exact when the poles of the mechanical system are confined to the upper half of the complex frequency plane. This is not the case for hysteretic damping models [25] and eq. (32) results into an approximation rather than an exact result. Nevertheless, the error quickly decreases when increasing the order of the filter, and it has been proven to be negligible compared to the error made by approximating a rectangular window by using a Butterworth filter [10]. When $n=1$, function $B$ corresponds to a Lorentzian function [26].

It is worth underlining the potential of the result in eq. (32). To evaluate the Butterworth-weighted frequency average, system displacements have to be computed at complex frequencies, which correspond to the positions of some of the poles of the Butterworth filter and no modification to the underlying numerical strategy is implemented. Moreover, for methods which require the solution of a system of complex matrices, like the WBM, this does not constitute an additional cost with respect to the evaluation at a real frequency. Finally, there is no dependence on the bandwidth over which the averaging is carried out. This means that the same number of function evaluations can be used regardless of the bandwidth, unlike numerical quadrature, of which the order has to be chosen as function of the interval of integration. In [10] it is shown that 8 to 10 function evaluations allow very accurate band computations. Due to the shape of the filter, using a smaller order has the effect of extending the average to frequencies outside the band.

It is worth mentioning that, at its current status, the use of the aforementioned strategy can only be efficiently exploited for input power (or input mobility) calculation, and it cannot be extended to quadratic quantities, like energy or sound power.

\section{Optimization procedures}

\subsection{Minimization of the power injected into the structure}

When dealing with the optimization of the dynamic behavior of a structure, it is of major interest to minimize the vibration. Depending on the 
problem, one may be interested in minimizing either the global or the local response. On the other hand, if the main concern is with the improvement of the noise emission, it may be of importance to minimize the acoustic power radiated from the structure.

The choice of the objective function plays a crucial role in the optimization process, and some computational aspects have to be taken into account. For example, using the acoustic radiated power, or any other acoustic-related quantity, as an objective function can lead to computationally demanding simulations. In fact, both structural and acoustic problems have to be solved for each iteration of the optimization process. This can significantly slow down the calculation time.

As an alternative to reduce the acoustic radiated power, Jog [27] suggests to minimize the power injected into the uncoupled structure. This strategy leads to a minimization of the input mobility of the structure. As a consequence, structural resonances are drifted away from the target frequency, and the overall level of vibration is decreased. Moreover, when the vibro-acoustic model is uncoupled, the optimization is performed on the bare structure, reducing the computational cost. The potential of this approach has been shown by means of several examples in [28].

When designing a DVA, the effects of damping loss mechanisms are strictly related to the injected power. In fact, the input power into the whole system is equal to the power dissipated by the host structure plus the power dissipated by the resonator. The former quantity is proportional to the average velocity of the host structure, while the latter is related to the difference between the velocity of the resonator and the one of the main system at the point where the device is located. The damping coefficients act like weightings for these contributions, and minimizing the power injected into the system leads to a reduction of the sum of the dissipated powers. Consequently, a reduction of the global velocity of the host system can be achieved, although in general, it is not minimized.

A delicate additional aspect to consider when designing a mechanical component is the frequency range over which the performance of the structure should be optimal. Usually this choice can be related to the knowledge of the external excitations. Moreover, the presence of uncertainty and variability may influence the nominal position of a resonance frequency, reducing the effectiveness of vibration control treatments. In these situations, optimizing the behavior over a band of frequencies is often preferred rather than targeting a single frequency. Nevertheless, the increased robustness of 
the configuration comes at a high computational price. In fact, the use of quadrature schemes to capture highly oscillating mobility functions can dramatically slow down the efficiency of the optimization scheme.

In this context, using the residue theorem to estimate band-averaged input power provides a powerful tool to perform accurate band evaluations at a reduced computational cost. This can efficiently be embedded in an optimization process and increase the overall performance of the procedure. Although in general this procedure does not lead to sound radiation or global vibration minimization, it can be very useful to quickly identify an improved design configuration at a very low computational cost. For this reason it can be easily employed for large applications.

\subsection{Genetic algorithms}

For this type of problems, GAs can be used [7, 29]. Unlike gradient-based optimization schemes, GAs are inspired by natural selection processes, where the best individuals dominate the population [30]. As a first step of the optimization scheme, a random population is created and ranked based on the fitness (objective function). Individuals with a higher ranking position have a higher probability of being selected for reproduction. The successive generation consists of children created by crossover, namely randomly mixing the selected parents. In this process the so-called mutation is also performed, by which the genetic sequence of an individual is mutated according to a random variable. The process is repeated until one of the convergence criteria is reached. The main advantage of evolutionary algorithms is their robustness, having an increased chance of finding a global optimum or near global optimum. On the other hand, they have a high computational cost.

The minimization of the input power, averaged over the interval $I$, can be formulated as a single objective ( $\mathrm{SO}$ ) optimization,

$$
\min \left\langle P_{\text {in }}\right\rangle_{I}
$$

subject to the side constraints,

$$
\underline{b}_{j} \leq b_{j} \leq \bar{b}_{j}, \quad j=1, \ldots, n_{\mathrm{var}},
$$

where each of the $n_{\text {var }}$ elements of the design variable vector, $b_{j}$, is defined by the upper and lower bounds, $\bar{b}_{j}$ and $\underline{b}_{j}$, respectively. During the design process, the engineer has the freedom to choose the variable $b_{j}$ with respect to which the input power is minimized. In the following examples, for each 
DVA, the location and the resonance frequency are the design variables to be optimized. Accordingly, the $x$ and $y$ coordinates are bounded by $\left[0, L_{x}\right]$ and $\left[0, L_{y}\right]$, respectively. For the resonance frequency, the bounds are much broader than the interval $I$, to allow the value to lie outside the target band. The choice of the damping also plays an important role as it influences the frequency band in which the energy is absorbed from the host system. Although in the following applications this is not explored, the damping of the DVA could be added as a design variable, according to the engineer's choice. Many optimization problems in engineering require the minimization of $n_{\mathrm{obj}}$ (conflicting) objectives. In this case, the problem is referred to as a multiobjective (MO) optimization and analogously to (33), it can be formulated as,

$$
\min \left[\left\langle P_{\text {in }}\right\rangle_{I_{1}},\left\langle P_{\text {in }}\right\rangle_{I_{2}}, \ldots,\left\langle P_{\text {in }}\right\rangle_{I_{n_{\text {obj }}}}\right]
$$

subject to the side constraints (34). Two main strategies exist to deal with MO problems $[31,32]$. According to the first category, the MO functions can be aggregated into a single function by means of a weighted sum, and the problem can be solved with SO optimization strategies. Here the choice of the weights plays a crucial role. Alternatively, it is often preferred to know the so-called Pareto front, which is the locus on which all the non-dominated solutions lie.

In the following application case, the first strategy is applied to solve a MO problem. The power injected into different bands is aggregated, weighted with equal weights and minimized by using GAs. However, the choice on the weights is in general not straightforward. In fact, if the power injected into one band is predominant with respect to other bands of interest, one could end up with a solution which attempts to minimize such a band and leave the others unaltered. Although this may be the best configuration, a different choice of the weighting factors may lead to a solution that is more effective for other bands.

\subsection{Summary and advantages of the procedure}

The aim of this section is to summarize the procedure adopted for the optimization and highlight the advantages in using the WBM in this context. Among all the properties of the WB modeling for vibration problems, two are of particular interest for this specific application. First of all, the WBM is a meshless approach. Consequently, it allows locating the resonator at any 
point over the plate and it does not require any re-meshing procedure. Using classic FEM would be much more disadvantageous, as the position of the DVA is confined to nodal points. Secondly, the WBM shows a higher convergence rate than element-based approaches, and a lower number of degrees of freedom is needed to model the problem. This becomes even more important when point connections are present, as they introduce a local perturbation, which requires a high mesh resolution to be properly captured. For point connections, another advantage is that the system of equations consists of a main part representing the bare system, $\mathbf{A}$ and $\mathbf{f}$, and a number of rows and columns, $\mathbf{B}, \mathbf{C}, \mathbf{D}$ and $\mathbf{f}^{\prime}$, of which the size is proportional to the number of point connections. Consequently, moving the position of a DVA or changing its properties does not require a change in the main system of equations.

To evaluate the input power over a band, only a few computations at complex frequencies are required. So, the small matrices of the bare system can be computed at the aforementioned complex frequencies and stored in memory. At each iteration they are recalled and complemented with the point connection contributions. The problem is solved for the wave contribution factors and the input power is computed.

GAs are used to solve the following optimization problem, that is performed in four steps:

1. Problem definition. Data related to the problem geometry, material, boundary conditions, excitation etc. are defined. Parameters of the GA are specified, i.e. tolerance, population size, stochastic parameters, etc.

2. Determination of the $n$ complex frequencies of analysis. According to the order of the Butterworth filter to use, the complex frequencies, at which the solution has to be computed, are determined,

$$
\mathbf{z}=\left[z_{1}, z_{2}, \ldots, z_{k}, \ldots, z_{n}\right]
$$

3. Evaluation and storage of system matrices. The WB matrices and right-hand side vectors for the bare system are computed at each complex frequency,

$$
\mathbf{A}_{1}, \mathbf{A}_{2}, \ldots, \mathbf{A}_{k}, \ldots, \mathbf{A}_{n}, \mathbf{f}_{1}, \mathbf{f}_{2}, \ldots, \mathbf{f}_{k}, \ldots, \mathbf{f}_{n} .
$$

Since $n$ is normally small and the WB matrices have a reduced number of DOFs, storing the matrices does not require large memory consumption. 
4. Optimization process. At each iteration, $l$, system matrices are recalled and complemented with a number of columns and rows equal to the number of point connections considered in the problem. Also the righthand side dimensions are increased,

$$
\left[\begin{array}{cc}
\mathbf{A}_{k} & \mathbf{B}_{k, l} \\
\mathbf{C}_{k, l} & \mathbf{D}_{k, l}
\end{array}\right]\left\{\begin{array}{c}
\mathbf{c}_{k, l} \\
\mathbf{w}_{\mathrm{pc}, l}
\end{array}\right\}=\left\{\begin{array}{c}
\mathbf{f}_{k} \\
\mathbf{f}_{k, l}^{\prime}
\end{array}\right\}
$$

the letter $k$ denotes the $k$-th complex frequency. Successively, the system of equations is solved and the input power is evaluated. This process is repeated until convergence is reached and function (32) is minimized.

The applicability of this strategy can easily be extended to a FE model. In fact, introducing a DVA at a certain point location involves the modification of the mass, stiffness and damping matrices of the corresponding node. Matrices can be computed only once and stored in memory. Within the iterative optimization procedure, the best configuration can be found. Although the WBM is more advantageous for this specific application, the proposed strategy can be used for cases where the potentialities of FEM are exploited at best, i.e. for complex geometries.

The algorithms applied here for the optimization process belong to the category of non-gradient based. Nevertheless, the procedure can be extended to gradient based algorithms, as shown by Koo [33]. In ref. [33], FEM is used to simulate the behavior of a vibrating cube, in which the thickness of a group of shell elements represents a set of design variables. The Adjoint Variable Method [34, 35] is used for the optimization process, while the residue theorem is used to evaluate the band-averaged power injected by harmonic point force excitations. This combination leads to a drastic abatement of the acoustic power radiated from the structure with a significant improvement in the computational performance.

\section{Application cases}

\subsection{Problem description}

The plate under consideration is simply supported and rectangular of dimensions $1 \times 0.6 \mathrm{~m}, 1 \mathrm{~mm}$ thick. The material is steel of Young modulus 210 GPa, density $7800 \mathrm{~kg} / \mathrm{m}^{3}$ and Poisson ratio 0.3 . The damping mechanism is hysteretic with constant loss factor equal to 0.01 . The plate is baffled and 
radiates in air characterized by a density of $1.225 \mathrm{~kg} / \mathrm{m}^{3}$, and a speed of sound of $340 \mathrm{~m} / \mathrm{s}$. The source is a harmonic point force arbitrarily chosen at $(0.85,0.15) \mathrm{m}$. The problem geometry is qualitatively depicted in fig. 1

The WBM is used to predict the behavior of the plate, and a truncation factor $T=2$ is chosen. To evaluate the acoustic radiated power, the Rayleigh approach is used, as described in eq. (10).

Two frequency ranges are of interest in the following examples. The first band covers the region from $26 \mathrm{~Hz}$ to $32 \mathrm{~Hz}$. Over that band, two modes are strongly contributing to the system response and they are located at 29.1 $\mathrm{Hz}(3,1)$ and $29.9 \mathrm{~Hz}(1,2)$. The second frequency range of interest sweeps frequencies from $58 \mathrm{~Hz}$ to $79 \mathrm{~Hz}$. In this case, four modes are contributing and two of them strongly radiate over the band. They are located at $64.1 \mathrm{~Hz}$ $(1,3), 66.9 \mathrm{~Hz}(4,2), 68.5 \mathrm{~Hz}(5,1)$ and $71.6 \mathrm{~Hz}(2,3)$. All the mode shapes are indicated in fig. 3 .

According to classic applications, the vibration level of the plate can be reduced by tuning the DVAs on structural resonances. This procedure leads to a reduction of the vibration at the target frequency, but it may result in a worse performance for the neighboring frequencies. To reduce this effect, a de-tuned configuration is often preferred, resulting into a mass-like or stiffness-like impedance of the resonator. This allows improving the vibrational behavior also for other frequencies. To further decrease the vibration level, several DVAs can be added to the host structure, especially when more than one mode is tackled. In the following applications, a reduced number of DVAs is used to tackle a band on which several modes are contributing. This serves as a pure example to show the potential of the present strategy for vibration control problems. However, also in this case, the number of DVAs could be increased to obtain superior noise and vibration reduction.

\subsection{Objective function evaluation and optimization}

Two types of optimization problems are carried out. The first one focuses on the minimization of the input power over the band $(58,79) \mathrm{Hz}$ as a SO optimization. The second one attempts to find the optimal design on the bands $(26,32) \mathrm{Hz}$ and $(58,79) \mathrm{Hz}$, using a MO optimization process. In all optimization processes, the design variables are the coordinates of the DVAs on the plate and their resonance frequencies. 
Different techniques are used to compute the objective function. Butterworth filters of order 4 (B4), 8 (B8) and 20 (B20) are used to evaluate the band-averaged power injected into the structure. Besides the number of computations, these schemes also differ in the weighting function. For a filter of order 4, a small weight is still assigned to frequencies outside the band, as shown in fig. 2. With increasing order, the shape of the filter becomes closer to that of a rectangular window. Alternatively, the trapezoidal scheme is used as a quadrature rule to estimate the band power. Three different integration steps are tested, namely $1 \mathrm{~Hz}$ (T1.0), $0.5 \mathrm{~Hz}$ (T0.5) and $0.2 \mathrm{~Hz}$ (T0.2). Finally, to compare the results provided by the input power minimization with more classic approaches, the space- and frequency-averaged displacement (DT0.5) and square velocity (V-T0.5) are also used as objective functions. In these cases, the trapezoidal rule with $0.5 \mathrm{~Hz}$ step is used to average over the frequency, while 1600 integration points are taken over the plate surface. In general, using classic quadrature schemes is more demanding, and the inaccuracy due to the rough integration step also influences the convergence of the optimization algorithm, as it will be shown in the following. Note that T1.0, T0.5 and T0.2 require 7, 13 and 31 function evaluations over the first band, and 22, 43 and 106 for the second band, respectively. By using the Butterworth-weighted frequency averaging, the function has to be evaluated only 4, 8 or 20 times according to the number of complex frequencies.

Regarding the optimization schemes, the objective is normalized with respect to the power injected into the bare plate, and the convergence is always reached when the average change in the fitness value is less than the tolerance $\left(10^{-7}\right)$ for 10 successive generations.

To quantify the reduction with respect to the initial design, a reduction coefficient, $r_{(\bullet)}$, is used and is computed by means of the following relation

$$
r_{(\bullet)}=100 \cdot \frac{\bullet_{\text {ini }}-\bullet_{\text {opt }}}{\bullet_{\text {ini }}},
$$

where $\bullet_{\text {ini }}$ stands for the initial value and $\bullet$ opt stands for the optimized value. Reduction coefficients refer to the band-averaged input power, acoustic radiated power and square averaged displacements. The averages are computed by using numerical quadrature with trapezoidal rule and $0.1 \mathrm{~Hz}$ sampling step.

All simulations are run on a Windows 7 64-bit desktop machine, processor Intel Core 2 Quad CPU, clock rate $3 \mathrm{GHz}$ and 8 GB RAM. GAs in Matlab are used to perform the optimization procedures. 


\begin{tabular}{|c|c|c|c|c|c|c||c|c|}
\hline Sim. ID & B4 & B8 & B20 & T1.0 & T0.5 & T0.2 & D-T0.5 & V-T0.5 \\
\hline$x_{p c}[m]$ & 0.879 & 0.879 & 0.878 & 0.878 & 0.878 & 0.878 & 0.870 & 0.869 \\
\hline$y_{p c}[m]$ & 0.124 & 0.124 & 0.125 & 0.123 & 0.125 & 0.125 & 0.137 & 0.138 \\
\hline$f_{\text {res }}[H z]$ & 85.9 & 85.9 & 86.1 & 86.7 & 86.0 & 86.1 & 72.4 & 71.5 \\
\hline$t_{\text {opt }}[s]$ & 198.0 & 406.0 & 1256.6 & 1590.7 & 2412.3 & 6059.9 & 4266.3 & 3039.0 \\
\hline Gen. $[-]$ & 41 & 44 & 55 & 62 & 46 & 48 & 51 & 36 \\
\hline$r_{\text {pow }}[-]$ & 74.0 & 74.0 & 74.0 & 74.0 & 74.0 & 74.0 & 70.3 & 69.0 \\
\hline$r_{\text {disp }}[-]$ & 92.5 & 92.5 & 92.2 & 92.0 & 92.3 & 92.2 & 95.6 & 95.9 \\
\hline$r_{\text {rad }}[-]$ & 88.4 & 84.4 & 87.9 & 87.2 & 88.0 & 87.9 & 95.0 & 95.0 \\
\hline
\end{tabular}

Table 1: Optimal configurations for SO optimization.

\subsection{Single objective optimization}

In this case, one DVA is used to reduce the vibration over the second band. The three design variables are the $x_{p c}$ and $y_{p c}$ position of the DVA on the plate and its resonance frequency, $f_{\text {res }}$. While the former variables are bounded according to the plate dimensions, the latter is bounded between 20 and $150 \mathrm{~Hz}$. The mass of the resonator is $2 \%$ of the mass of the bare plate, and the damping ratio is 0.1 . Regarding the GA settings, the population consists of 30 individuals.

The optimal configurations are reported in tab. 1, together with the total computation time, $t_{\mathrm{opt}}$, and the number of generations to reach convergence, Gen..

When the input power is minimized, all the optimization processes lead to similar designs. The resonator is located close to the excitation point, as it may be expected, and its resonance frequency falls above the interval of interest $(86.1 \mathrm{~Hz})$. It can be verified that this configuration minimizes the bandaveraged input power. All optimal designs present a noticeable improvement over the band. Data related to solving times show the computational advantages of the proposed approach, which are evident when comparing the design B4 with the ones obtained with more refined schemes. However, further gain in solving time can be achieved by adopting the strategy proposed in [11], which is based on similar principles.

Minimizing the space- and frequency-averaged displacement leads to configuration D-T0.5, reported in tab. 1, while V-T0.5 refers to the configuration that minimizes the averaged square velocity. Both configurations provide very close results, this is probably due to the fact that the targeted modes are very close to each other in frequency and thus minimizing the displace- 
ments and velocity leads to the similar optima. In these cases, the location of the resonator is not far from the one provided by the input power minimization. However, the resonance frequency for the D-T0.5 and V-T0.5 cases falls inside the frequency band under analysis in contrast to the B-series. The reduction of radiated power and averaged displacement of those configurations are higher than the ones of the B-series. This comes at a much higher computational cost, which becomes significant when comparing the performance of design B4 with D-T0.5 and V-T0.5. The improvements on the narrow band response can be observed in fig. 4, which compares designs B20 and DT0.5 in terms of input power, radiated acoustic power and averaged square displacement. The four peaks present on the narrow band input power are reduced over the frequency interval. This leads to strong improvements of the vibrational behavior of the panel. The configuration B20 presents a higher input power peak compared to the D-T0.5, which also leads to a higher radiated power and displacement level. However, as reported in tab. 1, the power injected over the band is smaller for the B20 configuration.

Figure 5 shows the local behavior of the frequency-averaged displacement over the plate for the initial configuration and the B20 design. The presence of the resonator allows reducing the overall vibration of the plate in an average sense.

To verify the robustness of the optimal configuration B20, the DVA parameters have been perturbed. The coordinates of the DVA can differ from their nominal values by $5 \mathrm{~mm}$; the resonance frequency of the DVA can differ by $1 \mathrm{~Hz}$; the mass by the $25 \%$ with respect to its nominal value; and the damping by $25 \%$ with respect to its nominal value. All parameters are uniformly distributed variables. Accordingly, a set of 300 random samples has been considered to compute the mean response and the upper and lower bounds of the input power, as shown in fig. 6. The deviation of the samples from the mean response is larger inside the band than for other frequencies. However, the perturbed design preserves improved characteristics compared to the initial one.

\subsection{Multi-objective optimization}

MO optimization is investigated in this example. As previously mentioned, there are two bands of interest, namely from 26 to $32 \mathrm{~Hz}$ and from 58 to $79 \mathrm{~Hz}$. Two DVAs are used to reduce the vibration. The mass of each device is equal to $0.1 \%$ of the mass of the bare plate, and their damping ratio is equal to 0.1. Six design variables are considered, corresponding to $x$ and 


\begin{tabular}{|c|c|c|c|c|c|c||c|c|}
\hline Simulation ID & B4 & B8 & B20 & T1.0 & T0.5 & T0.2 & D-T0.5 & V-T0.5 \\
\hline$x_{p c, 1}[\mathrm{~m}]$ & 0.502 & 0.503 & 0.505 & 0.722 & 0.701 & 0.505 & 0.504 & 0.502 \\
\hline$y_{p c, 1}[\mathrm{~m}]$ & 0.408 & 0.410 & 0.413 & 0.249 & 0.258 & 0.412 & 0.367 & 0.367 \\
\hline$f_{\text {res },[}[\mathrm{Hz}]$ & 29.9 & 29.5 & 29.5 & 59.5 & 62.1 & 29.2 & 28.8 & 28.9 \\
\hline$x_{p c, 2}[\mathrm{~m}]$ & 0.882 & 0.883 & 0.882 & 0.871 & 0.894 & 0.882 & 0.874 & 0.872 \\
\hline$y_{p c, 2}[\mathrm{~m}]$ & 0.121 & 0.123 & 0.124 & 0.131 & 0.118 & 0.122 & 0.135 & 0.134 \\
\hline$f_{\text {res }, 2}[\mathrm{~Hz}]$ & 79.9 & 80.2 & 80.2 & 81.4 & 84.6 & 80.2 & 70.9 & 70.0 \\
\hline$t_{\mathrm{opt}}[\mathrm{s}]$ & 747.6 & 1839.1 & 4420.2 & 1933.4 & 6808.6 & 13167.2 & 9388.5 & 10006.4 \\
\hline Gen. $[-]$ & 66 & 82 & 79 & 43 & 80 & 63 & 62 & 67 \\
\hline$r_{\text {pow }, 1}[-]$ & 30.2 & 30.6 & 30.5 & -2.9 & -1.9 & 30.7 & 22.4 & 22.2 \\
\hline$r_{\text {disp }, 1}[-]$ & 90.1 & 90.0 & 89.5 & -1.1 & -1.9 & 89.6 & 93.3 & 93.3 \\
\hline$r_{\text {rad }, 1}[-]$ & 87.5 & 88.1 & 87.9 & 8.7 & -3.9 & 88.4 & 89.7 & 89.4 \\
\hline$r_{\text {pow }, 2}[-]$ & 51.5 & 51.5 & 51.6 & 63.8 & 61.7 & 51.5 & 49.8 & 49.2 \\
\hline$r_{\text {disp }, 2}[-]$ & 86.7 & 86.4 & 86.3 & 92.7 & 91.7 & 86.4 & 92.3 & 92.4 \\
\hline$r_{\text {rad }, 2}[-]$ & 66.6 & 66.3 & 66.2 & 79.4 & 74.6 & 65.9 & 87.0 & 87.4 \\
\hline
\end{tabular}

Table 2: Optimal configurations for MO optimization.

$y$ positions and the resonance frequencies of the two resonators. Also in this case the resonance frequency variables are bounded by 20 and $150 \mathrm{~Hz}$. The GA population consists of 40 individuals.

The optimal configurations are shown in tab. 2, where the subscripts refer to the DVA when describing the location and resonance, and to the band for the reduction coefficients. Negative values in the reduction coefficients indicate that the optimal design is worse with respect to the initial one. This happens when rough trapezoidal quadrature schemes are not able to accurately represent the band value, and the optimization algorithm leads to a result which is not satisfactory. On the contrary, the residue theorem allows an accurate band evaluation, and results provided by the B-series do not differ much from each other. This explains why configurations T1.0 and T0.5 yield reduction coefficients $r_{\text {pow }}$, which are higher than the others on the second band, but much lower on the first one. In those configurations both DVAs tackle the same band.

In general, using the Butterworth-weighted frequency averaging procedure allows a significant speed up of the computation. This is clear when comparing configurations B4 and T0.2. In fact, although the optimal designs are not very different from each other, a speed up factor of about 17 can be observed. Also in this case, the minimization of the input power leads to a reduction of the radiated sound power and the averaged displacement over the plate. 
Although the lowest displacement level is not achieved in the B-series designs, reduction coefficients are not much lower than the D-T0.5 ones, especially on the first band. This can also be observed in fig. 7, where the narrow band responses of configurations B20 and D-T0.5 are illustrated. Conclusions similar to the ones for the previous example can be drawn for results on the band $(58,79) \mathrm{Hz}$, although the improvement is lower due to the reduced weight of the device in the current application. On the other hand, configurations B20 and D-T0.5 provide similar responses over the first band. Also in this case, minimizing the frequency- and space-averaged square velocity leads to a DVA configuration similar to the D-T0.5 case. From a computational point of view, the proposed approach highly outperforms the strategy adopted to design the D-T0.5 and V-T0.5 configurations, proving that it can be a very efficient tool for optimizing the DVA parameters in an early stage design, although, in principle, it does not guarantee the minimization of the velocity, but only its reduction.

In fig. 8 and 9, the frequency-averaged displacements are illustrated for the configuration B20. The first resonator tackles the modes present in the first band and is located close to the center of the plate. Its resonance falls inside the band of interest $(29.5 \mathrm{~Hz})$ and allows achieving a significant reduction of the overall displacement level. The second resonator is located close to the excitation point, like in the previous case, and its resonance frequency is above the band $(80.2 \mathrm{~Hz})$.

The robustness of configuration B20 against variability is assessed in fig. 10. The DVA parameters are perturbed like in the previous case: the coordinates of the DVA can differ from their nominal values by $5 \mathrm{~mm}$; the resonance frequency of the DVA can differ by $1 \mathrm{~Hz}$; the mass by the $25 \%$ with respect to its nominal value; and the damping by $25 \%$ with respect to its nominal value. Also in this case, all parameters are uniformly distributed. 300 random samples have been used to predict the ensemble behavior. Similarly to the previous example, over the bands of interest, the spread on the response is larger than for other frequencies. However, the perturbation does not worsen the overall performance of the optimal design, compared to the initial behavior of the system.

\section{Conclusions}

This paper presents a strategy to reduce the global vibration and radiated acoustic power, by minimizing the input power over a frequency band. The 
main feature of the present approach is the efficiency achieved by exploiting the residue theorem. Instead of requiring a refined quadrature integration, few calculations at complex frequencies allow accurate band computations, regardless of the bandwidth. This allows to significantly speed up the optimization process over frequency bands.

The strategy has been applied to optimize the properties of DVAs in order to improve the dynamic behavior of a baffled plate. In the first example, the properties of one DVA are optimized to minimize the power injected by a point force over a frequency band. In the second one, the characteristics of two DVAs are optimized to tackle two frequency bands. GAs have been used as an optimization tool in combination with the WBM, which shows high potential and efficiency for this application. For both examples, the optimization strategy has been proven to be very effective in reducing the radiated acoustic power and the overall displacement level over the band. A significant gain in computation time is achieved compared to classic schemes. Finally, the optimal configurations have been proven to be robust against DVA parameter variability.

Although in this paper the optimization strategy has been tested only on baffled plates, the underlying idea can be easily extended to more complex cases, i.e. complex geometries and different types of excitation, and to situations where robust and quick design is required.

\section{Acknowledgments}

The Fund for Scientific Research Flanders (F.W.O.), and the Research Fund KU Leuven are gratefully acknowledged for their support.

\section{References}

[1] J. Q. Sun, M. R. Jolly, M. A. Norris, Passive, Adaptive and Active Tuned Vibration Absorbers: A Survey, Journal of Mechanical Design 117 (1995) 234.

[2] J. P. Den Hartog, Mechanical Vibrations (Dover Civil and Mechanical Engineering), Dover Publications, 1985.

[3] J. Hunt, Dynamic Vibration Absorbers, Mechanical Engineering Publications, 1979. 
[4] Z. Sun, J. Sun, C. Wang, Y. Dai, Dynamic vibration absorbers used for increasing the noise transmission loss of aircraft panels, Applied Acoustics 48 (1996) 311-321.

[5] D. Rade, V. Steffen, Optimization of dynamic vibration absorbers over a frequency band, Mechanical Systems and Signal Processing 14 (2000) 679-690.

[6] N. Debnath, S. Deb, A. Dutta, Frequency band-wise passive control of linear time invariant structural systems with $h_{\infty}$ optimization, Journal of Sound and Vibration 332 (2013) 6044-6062.

[7] J. Holland, Adaptation in Natural and Artificial Systems: An Introductory Analysis with Applications to Biology, Control, and Artificial Intelligence, A Bradford Book, 1992.

[8] C. Q. Howard, C. H. Hansen, A. Zander, Vibro-acoustic noise control treatments for payload bays of launch vehicles: Discrete to fuzzy solutions, Applied Acoustics 66 (2005) 1235-1261.

[9] C. C. Claeys, K. Vergote, P. Sas, W. Desmet, On the potential of tuned resonators to obtain low-frequency vibrational stop bands in periodic panels, Journal of Sound and Vibration 332 (2013) 1418-1436.

[10] R. D'Amico, K. Koo, D. Huybrechs, W. Desmet, On the use of the residue theorem for the efficient evaluation of band-averaged input power into linear second-order dynamic systems, Journal of Sound and Vibration 332 (2013) 7205-7225.

[11] R. D'Amico, D. Huybrechs, W. Desmet, A refined use of the residue theorem for the evaluation of band-averaged input power into linear second-order dynamic systems, Journal of Sound and Vibration 333 (2014) 1796-1817.

[12] W. Desmet, A wave based prediction technique for coupled vibroacoustic analysis, Ph.D. thesis, KU Leuven, 1998.

[13] B. Pluymers, B. Hal, D. Vandepitte, W. Desmet, Trefftz-Based Methods for Time-Harmonic Acoustics, Archives of Computational Methods in Engineering 14 (2007) 343-381. 
[14] O. C. Zienkiewicz, The Finite Element Method, ButterworthHeinemann, $6^{\text {th }}$ edition, 2005.

[15] K. Vergote, Dynamic analysis of structural components in the mid frequency range using the wave based method: non-determinism and inhomogeneities, Ph.D. thesis, KU Leuven, 2012.

[16] L. Cremer, M. Heckl, B. Petersson, Structure-borne sound: Structural vibrations and sound radiation at audio frequencies, Springer, $3^{\text {rd }}$ edition edition, 2005.

[17] A. Leissa, Vibration of plates, Acoustical Society of America, 1993.

[18] M. Bruneau, Fundamental of Acoustics, ISTE Ltd., 2006.

[19] D. Hendy, Numerical evaluation of the sound power radiated from baffled, rectangular panels, Journal of Sound and Vibration 127 (1988) 283-289.

[20] E. Trefftz, Ein Gegenstück zum Ritzschen Vaerfahren, in: Proceedings of the Second International Congress on Applied Mechanics, Zurich, Switzerland.

[21] C. Vanmaele, D. Vandepitte, W. Desmet, An efficient wave based prediction technique for plate bending vibrations, Computer Methods in Applied Mechanics and Engineering 196 (2007) 3178-3189.

[22] K. Vergote, C. Vanmaele, D. Vandepitte, W. Desmet, An efficient wave based approach for the time-harmonic vibration analysis of 3D plate assemblies, Journal of Sound and Vibration 332 (2013) 1930-1946.

[23] P. Davis, P. Rabinowitz, Methods for numerical integration, Academic Press Inc., London, 1984.

[24] M. Greenberg, Advanced engineering mathematics, Pearson, $2^{\text {nd }}$ edition edition, 1998.

[25] M. Crane, J. Byrne, J. Glynn, J. Boyle, Causality and the Complex Modulus Model of Damping, Journal of Applied Mathematics and Mechanics 75 (1995) 807-808. 
[26] R. D'Amico, K. Vergote, R. Langley, W. Desmet, On the use of the Lorentzian function for the evalutaion of the frequency averaged input power into plates, in: Proceedings of the International Conference on Noise and Vibration (ISMA2012), Leuven, Belgium.

[27] C. Jog, Topology design of structures subjected to periodic loading, Journal of Sound and Vibration 253 (2002) 687-709.

[28] A. K. Nandy, C. S. Jog, Optimization of vibrating structures to reduce radiated noise, Structural and Multidisciplinary Optimization 45 (2012) $717-728$.

[29] D. Goldberg, Genetic Algorithms in Search, Optimization, and Machine Learning, Addison-Wesley Professional, 1989.

[30] G. Venter, Review of Optimization Techniques, Wiley Blackwell (John Wiley \&amp; Sons), 2010.

[31] P. Ngatchou, A. Zarei, A. El-Sharkawi, Pareto Multi Objective Optimization, Institute of Electrical and Electronics Engineers, pp. 84-91.

[32] E. Zitzler, M. Laumanns, S. Bleuler, A Tutorial on Evolutionary Multiobjective Optimization, Springer-Verlag, 2004.

[33] K. Koo, R. D’Amico, B. Pluymers, W. Desmet, Design optimization of the frequency averaged input power using the residue theorem, in: Proceedings of the $20^{\text {th }}$ International Congress on Sound and Vibration, ICSV20, Bangkok, Thailand.

[34] K. K. Choi, N.-H. Kim, Structural Sensitivity Analysis and Optimization 1: Linear Systems (Mechanical Engineering Series), Springer, 2005.

[35] K. Koo, B. Pluymers, W. Desmet, S. Wang, Vibro-acoustic design sensitivity analysis using the wave-based method, Journal of Sound and Vibration 330 (2011) 4340-4351. 


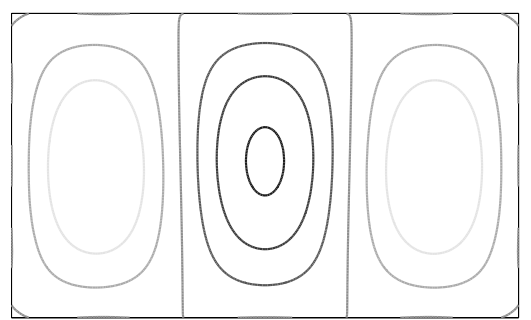

(a) $29.1 \mathrm{~Hz}$

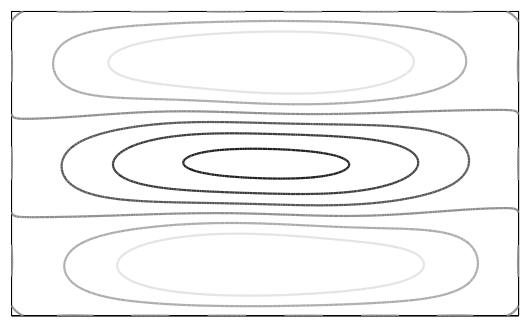

(c) $64.1 \mathrm{~Hz}$

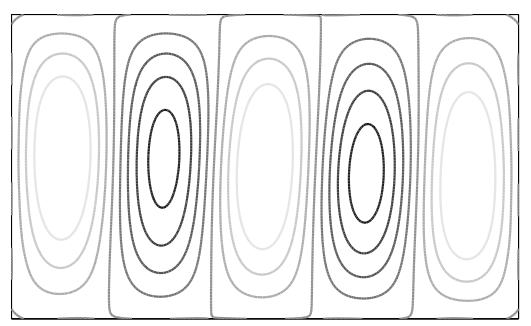

(e) $68.5 \mathrm{~Hz}$

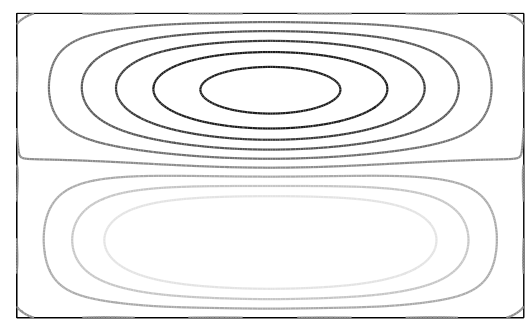

(b) $29.9 \mathrm{~Hz}$

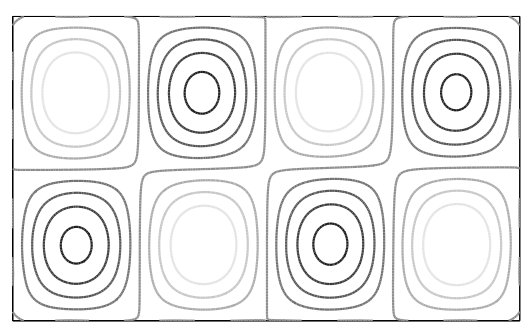

(d) $66.9 \mathrm{~Hz}$

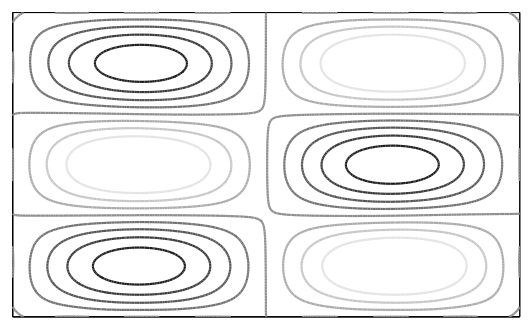

(f) $71.6 \mathrm{~Hz}$

Figure 3: Mode shapes inside the first band, (a) and (b), and the second band, (c) to (f). 

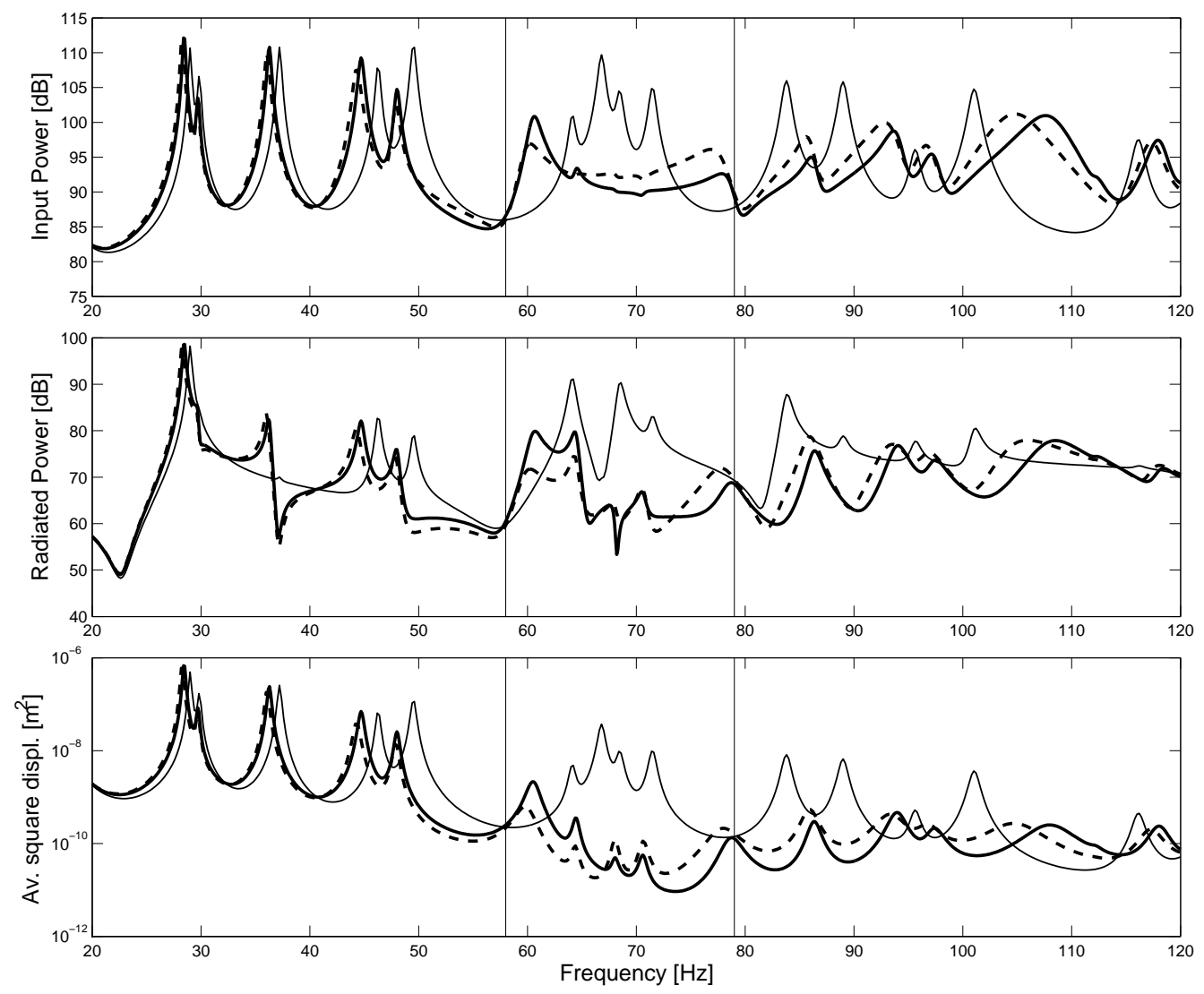

Figure 4: SO optimization. Initial configuration (thin solid line); B20 (thick solid line); D-T0.5 (dashed line). 

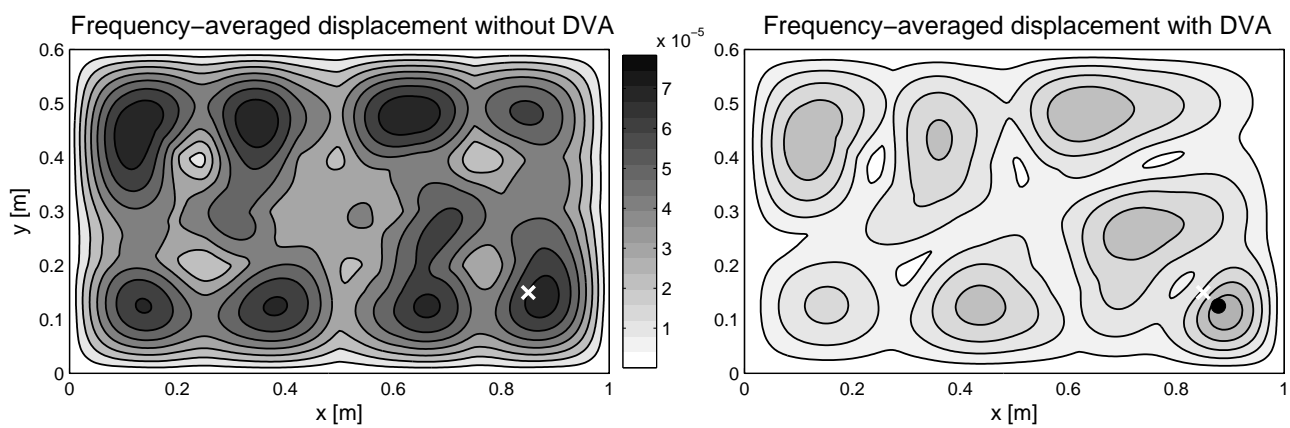

Figure 5: Contour plot of frequency-averaged displacement, $\langle|w|\rangle$, over the band $(58,79)$ Hz. The symbol $\times$ indicates the point force, while $\bullet$ the location of the DVA. The unit of the contour map is $[\mathrm{m}]$.

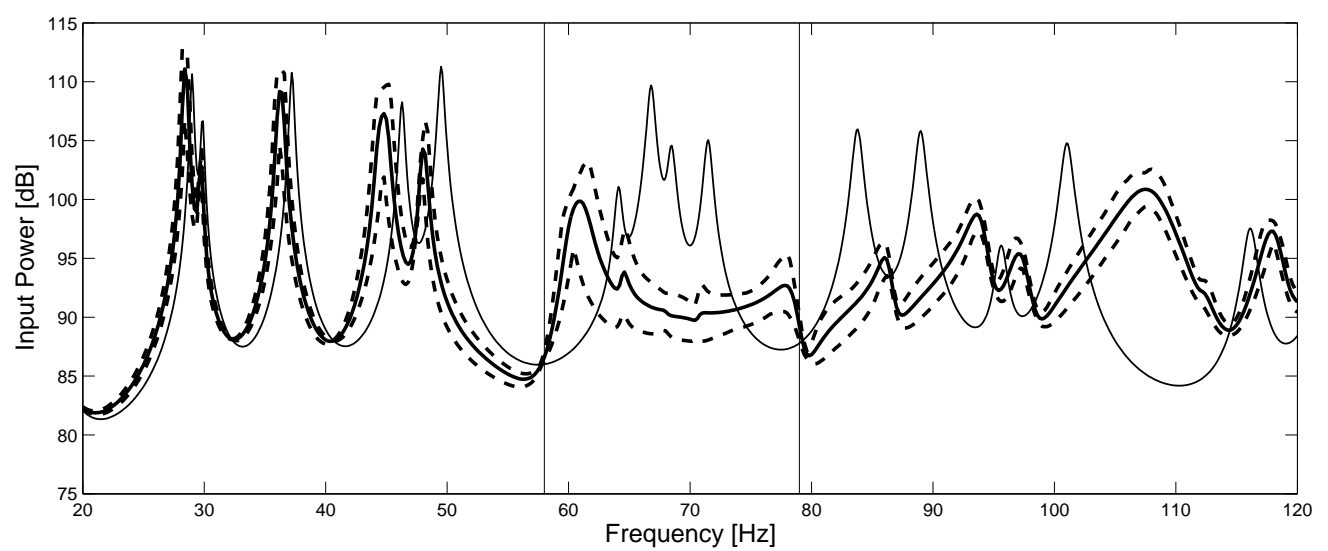

Figure 6: Effect of perturbation on DVA properties for the case of SO optimization. Initial configuration (thin solid line); ensemble average (thick solid line); upper and lower bounds of the ensemble (dashed lines). 

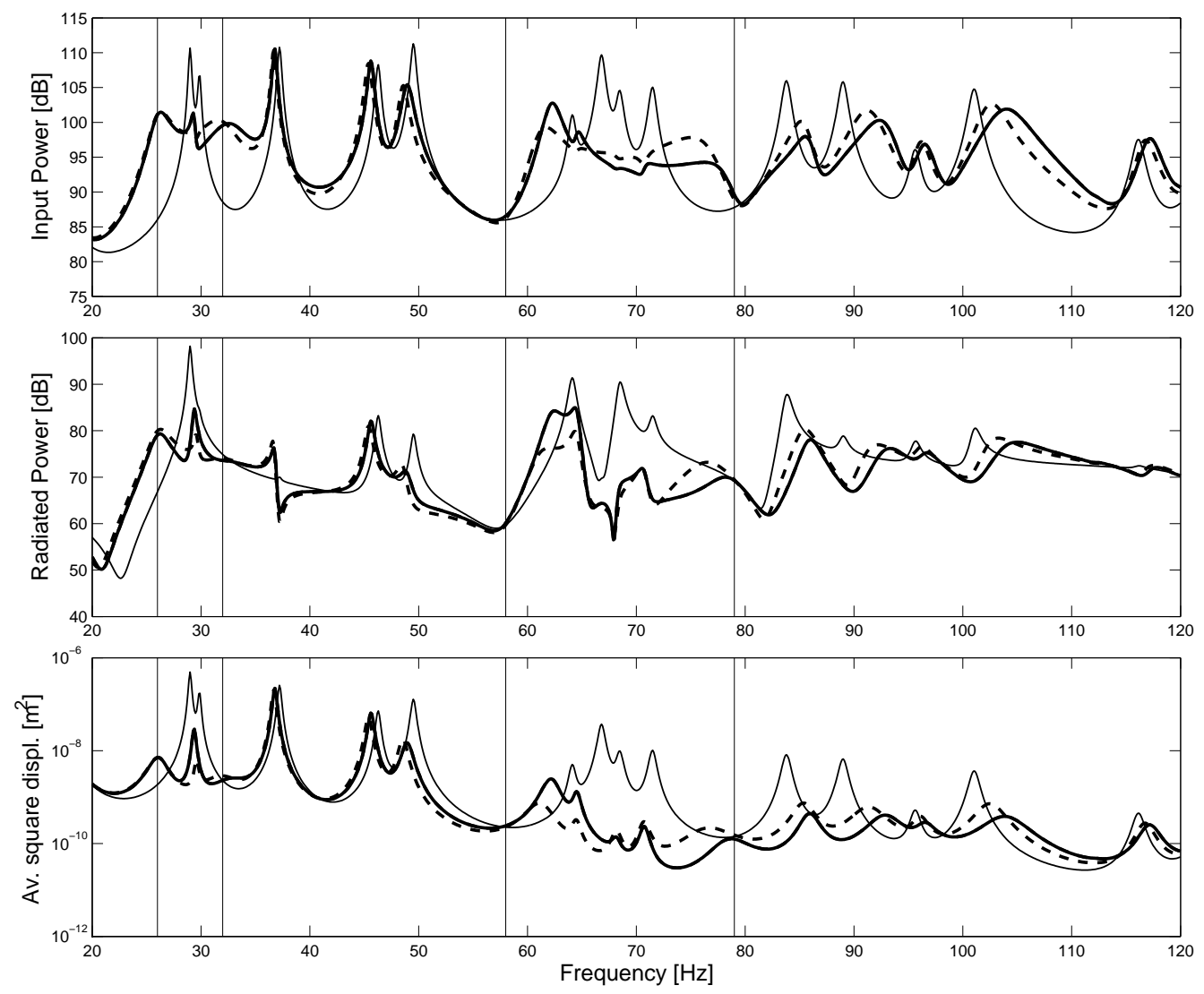

Figure 7: MO optimization. Initial configuration (thin solid line); B20 (thick solid line); D-T0.5 (dashed line). 

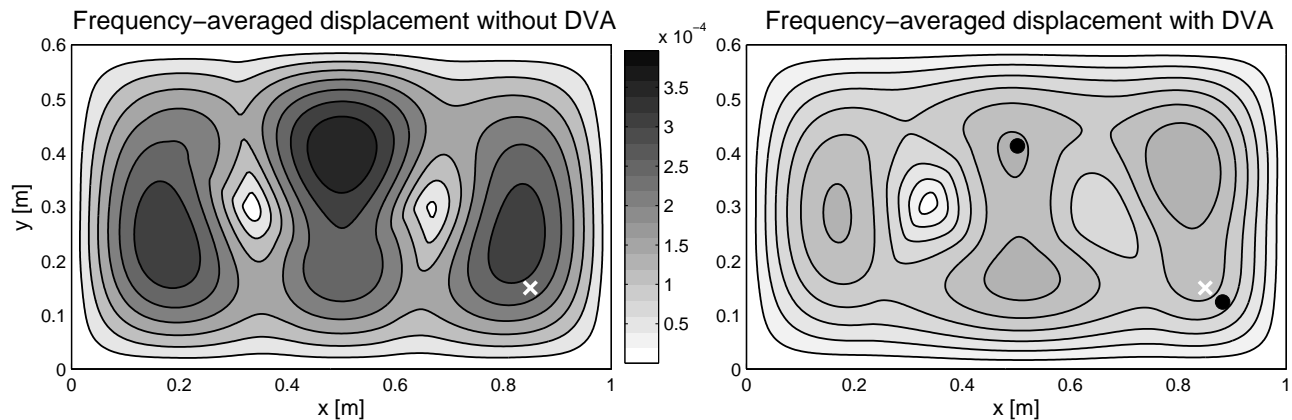

Figure 8: Contour plot of frequency-averaged displacement, $\langle|w|\rangle$, over the band $(26,32)$ Hz. The symbol $\times$ indicates the point force, while $\bullet$ the location of the DVA. The unit of the contour map is $\mathrm{m}$.
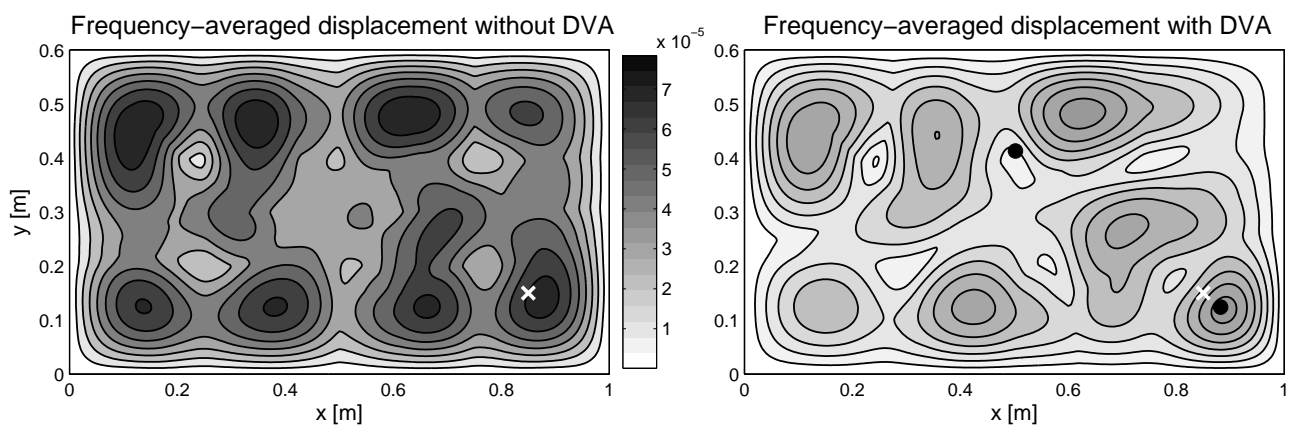

Figure 9: Contour plot of frequency-averaged displacement, $\langle|w|\rangle$, over the band $(58,79)$ Hz. The symbol $\times$ indicates the point force, while $\bullet$ the location of the DVA. The unit of the contour map is $\mathrm{m}$. 


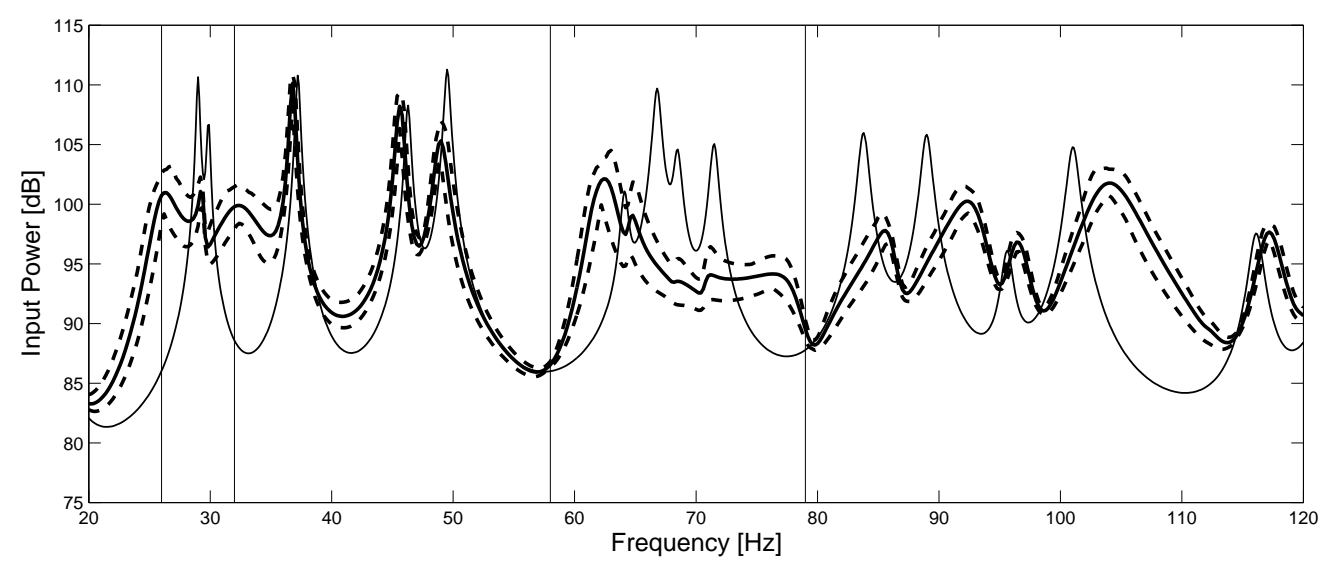

Figure 10: Effect of perturbation on DVA properties for the case of MO optimization. Initial configuration (thin solid line); ensemble average (thick solid line); upper and lower bounds of the ensemble (dashed lines). 